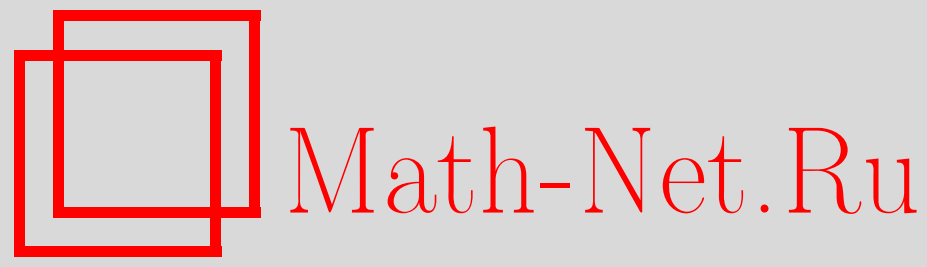

П. К. Розанов, Об экспоненциальности аффинных симметрических пространств, Функи. анализ и его прил., 2009, том 43, выпуск 1, 68-80

DOI: https://doi.org/10.4213/faa2939

Использование Общероссийского математического портала Math$\mathrm{Net.Ru} \mathrm{подразумевает,} \mathrm{что} \mathrm{вы} \mathrm{прочитали} \mathrm{и} \mathrm{согласны} \mathrm{с} \mathrm{пользователь-}$ ским соглашением

http://www . mathnet.ru/rus/agreement

Параметры загрузки:

IP : 3.80 .181 .102

26 апреля 2023 г., 17:26:09

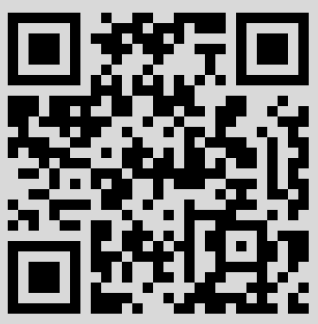


Функциональный анализ и его приложения

2009, т. 43, вып. 1, с. $68-80$

УДК 512.816.4

\title{
Об экспоненциальности аффинных симметрических пространств
}

\author{
(C) 2009. П. K. РозАнов
}

\begin{abstract}
Аффинное симметрическое пространство вида $G / H$ называется экспоненциальным, если любые две его точки можно соединить геодезической, и слабо экспоненциальным, если объединение всех геодезических, проведенных из одной точки, всюду плотно. Для группового пространства $(G \times G) / G_{\text {diag }}$ группы Ли $G$ эти свойства эквивалентны соответственно экспоненциальности и слабой экспоненциальности группы $G$. В работе известные теоремы об образе экспоненциального отображения в группах Ли обобщаются на случай аффинных симметрических пространств. Доказывается слабая экспоненциальность симметрических пространств разрешимых групп Ли, а для полупростого случая получены критерии экспоненциальности и слабой экспоненциальности.
\end{abstract}

\section{§1. Введение}

Группа Ли $G$ называется слабо экспоненииалъной, если $G=\overline{\exp \mathfrak{g}}$, где $\mathfrak{g}=$ $\operatorname{Lie} G$, и экспоненциальной, если $G=\exp \mathfrak{g}$. Алгебра Ли $\mathfrak{g}$ называется (слабо) экспоненциальной, если существует (слабо) экспоненциальная группа Ли с касательной алгеброй $\mathfrak{g}$. Хорошо известно, что всякая связная компактная группа Ли экспоненциальна. Хоффман и Мухерджи [8] показали, что любая связная разрешимая и любая связная комплексная группы Ли слабо экспоненциальны. Ими же доказано, что слабая экспоненциальность произвольной группы Ли эквивалентна слабой экспоненциальности ее фактора по радикалу, что сводит исследование слабой экспоненциальности к полупростому случаю. Нееб [12] получил критерий слабой экспоненциальности произвольной группы Ли, а Вюстнер [15] - критерий экспоненциальности расщепимых групп Ли и их накрытий (линейная группа Ли называется расщепимой, если содержит компоненты разложения Жордана каждого своего элемента). В частности, критерий Вюстнера применим к полупростым группам Ли. K настоящему времени получены классификации слабо экспоненциальных и экспоненциальных простых алгебр Ли (см. обзор [7]), а также экспоненциальных простых групп Ли ([10], [16]).

В этой статье будут рассмотрены обобщения перечисленных выше результатов на аффинные симметрические пространства.

Введем основные обозначения. Пусть $G-$ связная вещественная группа Ли, $\sigma$ - ее инволютивный автоморфизм и $G^{\sigma} \subset G-$ подгруппа элементов, неподвижных относительно автоморфизма $\sigma$. Для произвольной группы Ли $L$ обозначим через $L^{0}$ ее компоненту связности, содержащую единицу. Аффиннъцм симметрическим пространством называется однородное пространство вида $X=G / H$, где $H \subset G$ - подгруппа Ли, удовлетворяющая условию $\left(G^{\sigma}\right)^{0} \subseteq$ $H \subseteq G^{\sigma}$. Касательная алгебра $\mathfrak{g}=\operatorname{Lie} G$ разлагается в прямую сумму собственных пространств автоморфизма $\mathrm{d} \sigma: \mathfrak{g}=\mathfrak{h}+\mathfrak{m}$, где

$$
\mathfrak{h}=\text { Lie } H=\{\xi \in \mathfrak{g}: \mathrm{d} \sigma(\xi)=\xi\}, \quad \mathfrak{m}=\{\xi \in \mathfrak{g}: \mathrm{d} \sigma(\xi)=-\xi\} .
$$


Обозначим через $M_{G}$ связную компоненту множества $\left\{g \in G: \sigma(g)=g^{-1}\right\}$, содержащую единицу. Множество $M_{G}$ является гладким подмногообразием в $G$. Действительно, фиксируем такую окрестность нуля $U \subset \mathfrak{g}$, что ограничение экспоненциального отображения на нее является диффеоморфизмом, и для произвольного элемента $g \in M_{G}$ рассмотрим его окрестность в $G$ вида $W_{g}=g \exp U_{1}$, где $U_{1}=U \cap \operatorname{Ad}\left(g^{-1}\right) U \cap \mathrm{d} \sigma(U)$. Отображение $f_{g}: U_{1} \rightarrow W_{g}, f_{g}(\xi)=g \exp \xi,-$ диффеоморфизм. Пересечение $M_{G} \cap W_{g}$ имеет вид $\left\{f_{g}(\xi): \xi \in U_{1},(\operatorname{Ad}(g)+\right.$ $\mathrm{d} \sigma)(\xi)=0\}$, т. е. является образом пересечения некоторого линейного подпространства касательной алгебры $\mathfrak{g}$ с окрестностью $U_{1}$. Очевидно, что $\exp \mathfrak{m} \subseteq$ $M_{G}$, причем множество $\exp \mathfrak{m}$ содержит некоторую окрестность единицы в $M_{G}$.

На аффинном симметрическом пространстве $X=G / H$ можно ввести каноническую $G$-инвариантную линейную связность. При этом геодезическими будут орбиты однопараметрических групп вида $\exp \langle\xi\rangle, \xi \in \mathfrak{m}$. Назовем пространство $X$ экспоненииальным, если любые две его точки можно соединить геодезической, и слабо экспоненииалъным, если объединение всех геодезических, проведенных из некоторой точки, всюду плотно в $X$. В терминах группы $G$ экспоненциальность эквивалентна условию $G=(\exp \mathfrak{m}) H$, а слабая экспоненциальность - условию $G=\overline{(\exp \mathfrak{m}) H}$. Для любого симметрического пространства $G / H$ существует такая группа Ли $G^{\prime}$, накрывающая группу $G$, что автоморфизм $\sigma$ поднимается до автоморфизма группы $G^{\prime}$ и полный прообраз группы $H$ при отображении накрытия совпадает с группой $\left(G^{\prime}\right)^{\sigma}[13$, лемма 3]. Поэтому при исследовании экспоненциальности и слабой экспоненциальности можно ограничиться рассмотрением пространств вида $G / G^{\sigma}$.

Группу Ли $G$ можно рассматривать как симметрическое пространство $(G \times$ $G) / G_{\text {diag }}$, где $G_{\text {diag }}=\{(g, g): g \in G\}$ - множество неподвижных точек автоморфизма $\sigma$, переставляющего компоненты прямого произведения. (Слабая) экспоненциальность группы $G$ как симметрического пространства эквивалентна ее (слабой) экспоненциальности как группы Ли.

Известно, что всякое риманово симметрическое пространство экспоненциально. Однако для произвольного аффинного симметрического пространства это неверно.

Пример 1. Рассмотрим полупрямое произведение $G=\mathbb{R} \wedge_{\alpha} \mathbb{C}$, где гомоморфизм $\alpha: \mathbb{R} \rightarrow \operatorname{Aut}(\mathbb{C})$ задается формулой $\alpha(t) c=e^{i t} c, t \in \mathbb{R}, c \in \mathbb{C}$, и инволютивный автоморфизм $\sigma(t, c)=(-t, \bar{c})$ группы $G$. В этом случае множество $G \backslash(\exp \mathfrak{m}) G^{\sigma}$ имеет вид $\{(t, c): t \in 2 \pi(\mathbb{Z} \backslash\{0\}, \operatorname{Im} c \neq 0\}$. Оно непусто, но является подмногообразием коразмерности 1 в $G$. Следовательно, пространство $G / G^{\sigma}$ слабо экспоненциально, но не экспоненциально.

Другим примером неэкспоненциального симметрического пространства может служить групповое пространство неэкспоненциальной группы Ли, например, $\operatorname{SL}(2, \mathbb{R})$.

Основные результаты статьи заключаются в следующих четырех теоремах.

Теорема 1. Если группа $G$ разрешима, то пространство $G / G^{\sigma}$ слабо экспоненииально.

Обозначим через $\operatorname{Rad}(G)$ радикал группы $G$, т. е. ее максимальную связную разрешимую нормальную подгруппу. Эта подгруппа $\sigma$-инвариантна, так что автоморфизм $\sigma$ корректным образом индуцирует инволютивный автоморфизм факторгруппы $G / \operatorname{Rad}(G)=\widehat{G}$, который также будет обозначаться через $\sigma$. 
Теорема 2. Пространство $G / G^{\sigma}$ слабо экспоненциально тогда и только тогда, когда пространство $\widehat{G} / \widehat{G}^{\sigma}$ слабо экспоненциально.

С помощью теорем 1 и 2 исследование слабой экспоненциальности симметрических пространств сводится к полупростому случаю. Для формулировки критерия слабой экспоненциальности в этом случае нам понадобится понятие картановской группы симметрического пространства.

Пусть $G$ - редуктивная линейная группа Ли. Картановской алгеброй пространства $G / G^{\sigma}$ называется максимальная коммутативная подалгебра $\mathfrak{a} \subset \mathfrak{m}$, состоящая из полупростых элементов. Картановской группой пространства $G / G^{\sigma}$, соответствующей картановской алгебре $\mathfrak{a}$, называется группа $Z_{M_{G}}(\mathfrak{a})=$ $Z_{G}(\mathfrak{a}) \cap M_{G}$. Всякая картановская группа коммутативна [13]. Фиксируем инволюцию Картана $\theta$, коммутирующую с автоморфизмом $\sigma$, и рассмотрим соответствующее разложение Картана $\mathfrak{g}=\mathfrak{k}+\mathfrak{p}$, где $\mathfrak{k}-$ компактная часть, а $\mathfrak{p}-$ некомпактная. Назовем $\theta$-инвариантную картановскую алгебру $\mathfrak{a}$ и соответствующую ей картановскую группу максимально некомпактными, если пересечение $\mathfrak{a} \cap \mathfrak{k}$ имеет минимальную размерность. Максимально некомпактная картановская группа пространства $G / G^{\sigma}$ единственна с точностью до сопряжения при помощи $G^{\sigma}$.

Теорема 3. Пусть $G$ - редуктивная линейная группа Ли. Тогда следующие условия эквивалентны:

1) симметрическое пространство $G / G^{\sigma}$ слабо экспоненииально;

2) любая картановская группа пространства $G / G^{\sigma}$ связна;

3) максимально некомпактная картановская группа пространства $G / G^{\sigma}$ связна.

Теорема 4. Если $G$ - расщепимая линейная группа Ли, то пространство $G / G^{\sigma}$ экспоненциально тогда и только тогда, когда для любого нильпотентного элемента $\xi \in \mathfrak{m}$ множество $Z_{G}(\xi) \cap M_{G}$ свлзно и пространство $Z_{G}(\xi)^{0} /\left(Z_{G}(\xi)^{0}\right)^{\sigma}$ слабо экспоненииально.

Замечание. Пусть $G$ - линейная полупростая группа Ли. Тогда она расщепима. Обозначим через $S(\xi) \sigma$-инвариантную редуктивную подгруппу Леви группы $Z_{G}(\xi)^{0}$. Известно, что $S(\xi)=Z_{G}(\mathfrak{l}(\xi))^{0}$, где $\mathfrak{l}(\xi) \subset \mathfrak{g}$ есть $\mathfrak{s l}(2, \mathbb{R})$-тройка, содержащая элемент $\xi$. По теореме 2 слабая экспоненциальность пространства $Z_{G}(\xi)^{0} /\left(Z_{G}(\xi)^{0}\right)^{\sigma}$ эквивалентна слабой экспоненциальности пространства $S(\xi) / S(\xi)^{\sigma}$, при исследовании которой можно воспользоваться теоремой 3.

В случае группового пространства теоремы 1-4 эквивалентны известным результатам об образе экспоненциального отображения группы Ли. Результаты, соответствующие теоремам 1 и 2, были получены Хоффманом и Мухерджи [8], а теоремы 3 и 4 обобщают критерии слабой экспоненциальности и экспоненциальности для групп Ли, принадлежащие соответственно Неебу [12] и Вюстнеру [15].

\section{§2. Предварительные результаты}

Определим действие группы $G$ на себе по следующему правилу: $g \cdot h=$ $g h \sigma(g)^{-1}$. Легко видеть, что множество $M=M_{G}$ инвариантно относительно этого действия. Орбита единицы $G \cdot e=\left\{g \sigma(g)^{-1}: g \in G\right\}$ содержит множество exp $\mathfrak{m}$ и, следовательно, открыта в $M$. 
Предложение 5. Симметрическое пространство $X=G / G^{\sigma}$ слабо экспоненциально тогда и только тогда, когда $G \cdot e=\overline{\exp \mathfrak{m}}$, и экспоненциально тогда u только тогда, когда $G \cdot e=\exp \mathfrak{m}$.

Доказательство. Отображение $f: X \rightarrow G \cdot e, f\left(g G^{\sigma}\right)=g \sigma(g)^{-1}$, является диффеоморфизмом. Образом геодезической $\exp \langle\xi\rangle G^{\sigma}, \xi \in \mathfrak{m}$, при этом отображении будет однопараметрическая подгруппа $\exp \langle\xi\rangle$. Отсюда легко следует доказываемое утверждение.

Назовем группу Ли $G$ сильно экспоненциальной, если ее экспоненциальное отображение является диффеоморфизмом (иногда под экспоненциальностью понимают именно это свойство, см., например, [1]). Ясно, что всякая сильно экспоненциальная группа Ли должна быть односвязной. Одним из известных критериев сильной экспоненциальности является следующая теорема:

Теорема 6 [1, с. 67, теорема 6.4]. Односвязная группа Ли сильно экспоненииалъна тогда и только тогда, когда присоединенный оператор любого элемента ее касательной алгебры не имеет ненулевых чисто мнимых собственных значений.

В частности, любая односвязная нильпотентная группа Ли сильно экспоненциальна.

Предложение 7. Любое симметрическое пространство силъно экспоненциальной группь Ли $G$ экспоненциально.

Доказательство. Так как группа $G$ односвязна, то подгруппа неподвижных точек любого ее инволютивного автоморфизма связна и любое симметрическое пространство группы $G$ имеет вид $G / G^{\sigma}$. Для любого $g \in M$ найдется такой $\xi \in \mathfrak{g}$, что $g=\exp \xi$. Но тогда из равенств $\exp (-\xi)=g^{-1}=\sigma(g)=\exp \mathrm{d} \sigma(\xi)$ и сильной экспоненциальности группы $G$ следует, что $\mathrm{d} \sigma(\xi)=-\xi$. Таким образом, $M=\exp \mathfrak{m}=G \cdot e$ и пространство $G / G^{\sigma}$ экспоненциально.

Теорема 8. Действие $G: M$ транзитивно.

Так как орбита единицы открыта в $M$, необходимо доказать только ее замкнутость. Для этого мы воспользуемся критерием Луны [2, с. 249, теорема 6.17]:

Теорема 9 (критерий Луны). Пусть редуктивная комплексная алгебраическая группа $G$ действует на комплексном афбинном многообразии $X$, и пусть $H$ - редуктивная подгруппа в $G, a x$ - некоторая точка из $X^{H}$. Для того чтобы орбита $G x$ была замкнутой, необходимо и достаточно, чтобъ бъла замкнутой орбита $N_{G}(H) x$.

Пусть $N$ есть $\sigma$-инвариантная нормальная подгруппа группы $G$, а $\pi: G \rightarrow$ $\widehat{G}=G / N-$ канонический гомоморфизм, $\pi(g)=g N$. Автоморфизм $\sigma$ индуцирует инволютивный автоморфизм группы $\widehat{G}$, который мы также обозначим через $\sigma$. Положим $\widehat{M}=M_{\widehat{G}}$ и рассмотрим действие $\widehat{G}: \widehat{M}$, аналогичное определенному ранее для группы $G$.

Лемма 10. Если подгруппа $N$ дискретна, то действие $G: M$ транзитивно тогда и только тогда, когда транзитивно действие $\widehat{G}: \widehat{M}$.

Доказательство. Прежде всего, докажем, что $\pi(M)=\widehat{M}$. Включение $\pi(M)$ $\subseteq \widehat{M}$ очевидно. Пусть $\hat{g} \in \widehat{M}$. Так как множество $\widehat{M}$ линейно связно, существует такой непрерывный путь $\hat{\gamma}(t) \subset M, 0 \leqslant t \leqslant 1$, что $\hat{\gamma}(0)=e, \hat{\gamma}(1)=\hat{g}$. Он единственным образом поднимается до такого пути $\gamma$ в $G$, что $\pi \circ \gamma=\hat{\gamma}$ и 
$\gamma(0)=e$. Пусть $\gamma(1)=g$. Для любого $t \in[0 ; 1]$ элемент $n(t)=\gamma(t) \sigma(\gamma(t))$ лежит в $N$. Так как группа $N$ дискретна, то для любого $t \in[0 ; 1]$ верно равенство $n(t)=n(0)=e$. Следовательно, $g \in M$ и $\hat{g} \in \pi(M)$.

Ясно, что гомоморфизм $\pi$ отображает орбиты группы $G$ в орбиты группы $\widehat{G}$. Таким образом, если действие $G: M$ транзитивно, то транзитивно и действие $\widehat{G}: \widehat{M}$. Обратно, если у действия $\widehat{G}: \widehat{M}$ одна орбита, то ее полный прообраз в $M$ распадается на орбиты одинаковой размерности. Но множество $M$ связно и содержит открытую орбиту. Следовательно, $G \cdot e=M$.

Лемма 11. Если подгруппа $N$ сильно экспоненциальна, то из транзитивности действия $\widehat{G}: \widehat{M}$ следует транзитивность действия $G: M$.

Доказательство. Фиксируем $x \in M$. Так как $\pi(x) \in \widehat{M}=\widehat{G} \cdot e$, то найдется такой $h \in G$, что $x_{1}=h \cdot x \in M \cap N$. Но тогда $x_{1} \in \exp (\mathfrak{m} \cap \mathfrak{n}) \subseteq G \cdot e$. Следовательно, $x \in G \cdot e$.

Доказательство теоремы 8. В силу леммы 10 достаточно рассмотреть случай, когда группа $G$ односвязна. Рассмотрим разложение Леви $\mathfrak{g}=\mathfrak{r}+\mathfrak{s}$, где $\mathfrak{r}$ - радикал алгебры $\mathfrak{g}$, а $\mathfrak{s}$ - полупростая подалгебра. Известно [4, с. 135, предложение 5], что $[\mathfrak{r}, \mathfrak{g}]=\mathfrak{u}$, где $\mathfrak{u}-$ нильпотентный радикал алгебры $\mathfrak{g}$. Следовательно, коммутант $[\mathfrak{g}, \mathfrak{g}]$ имеет вид $\mathfrak{u}+\mathfrak{s}$ (полупрямая сумма). Пусть $U$ - связная нормальная подгруппа группы $G$ с касательной алгеброй $\mathfrak{u}$. Так как группа $G$ односвязна, то $U$ является подгруппой Ли, причем она $\sigma$-инвариантна и односвязна, а значит, сильно экспоненциальна. По лемме 11 нам достаточно доказать утверждение теоремы для факторгруппы $G / U$, являющейся прямым произведением односвязной полупростой группы Ли на векторную группу Ли. Наконец, еще раз воспользовавшись леммой 11, мы можем считать, что группа $G$ полупроста.

По лемме 10 нам достаточно рассмотреть случай, когда $G$ - вещественная форма односвязной комплексной полупростой группы Ли. Автоморфизм $\sigma$ продолжается до автоморфизма комплексификации $G(\mathbb{C})$. При этом $G(\mathbb{C})$ - комплексная алгебраическая группа, а ее подгруппа $G(\mathbb{C})^{\sigma}$ связна и редуктивна. Применим критерий Луны к действию группы $G(\mathbb{C})$ на аффинном многообразии $M_{G(\mathbb{C})}$. Так как $\left(N_{G(\mathbb{C})}\left(G(\mathbb{C})^{\sigma}\right)\right)^{0}=G(\mathbb{C})^{\sigma}$, то орбита $N_{G(\mathbb{C})}\left(G(\mathbb{C})^{\sigma}\right) \cdot e$ конечна, а значит, орбита $G(\mathbb{C}) \cdot e$ замкнута в $M_{G(\mathbb{C})}$ по критерию Луны. Следовательно, $G(\mathbb{C}) \cdot e=M_{G(\mathbb{C})}$. Рассмотрим какой-нибудь $x \in M$. Образ касательного пространства орбиты $G \cdot x$ при правом сдвиге на элемент $x^{-1}$ есть $\{\eta-\operatorname{Ad}(x) \mathrm{d} \sigma(\eta)$ : $\eta \in \mathfrak{g}\} \subset \mathfrak{g}$, и его размерность равна размерности $\operatorname{dim}_{\mathbb{C}}(G(\mathbb{C}) \cdot x)$. Но это число одинаково для всех $x \in M$, так как $M \subset M_{\mathbb{C}}$. Поэтому размерности любых двух орбит действия группы $G$ на многообразии $M$ равны, а значит, орбита только одна.

Другое доказательство замкнутости орбиты единицы для действия $G: M$ в случае, когда группа $G$ полупроста, можно найти в статье [13] (лемма 1 ).

Следствие 12. Симметрическое пространство $G / G^{\sigma}$ слабо экспоненциально тогда и только тогда, когда $M=\overline{\exp \mathfrak{m}}$, и экспоненииально тогда и только тогда, когда $M=\exp \mathfrak{m}$.

Пусть $N$ - произвольная связная $\sigma$-инвариантная нормальная подгруппа группы $G, \widehat{G}=G / N$. Из теоремы 8 следует, что $\pi(M)=\widehat{M}$. 
Предложение 13. Пусть симметрическое пространство $G / G^{\sigma}$ (слабо) экспоненциально. Тогда (слабо) экспоненциально и пространство $\widehat{G} / \widehat{G}^{\sigma}$.

Доказательство. Пусть $\hat{g} \in \widehat{M}, g \in \pi^{-1}(\hat{g})$. Если существует такой $\xi \in \mathfrak{m}$, что $\exp \xi=g$, то $\exp (\mathrm{d} \pi(\xi))=\pi(\exp \xi)=\hat{g}$. Поэтому из экспоненциальности пространства $G / G^{\sigma}$ следует экспоненциальность пространства $\widehat{G} / \widehat{G}^{\sigma}$.

Теперь пусть пространство $G / G^{\sigma}$ слабо экспоненциально, и пусть $U$ - некоторое открытое подмножество множества $\widehat{M}$. Прообраз $\pi^{-1}(U)$ открыт в $M$, следовательно, существует такой $\xi \in \mathfrak{m}$, что $\exp \xi \in \pi^{-1}(U)$. Тогда $\exp (\mathrm{d} \pi(\xi))=$ $\pi(\exp \xi) \in U$.

\section{§3. Слабая экспоненциальность симметрических пространств разрешимых групп Ли}

В этом параграфе будут доказаны теоремы 1 и 2, сформулированные во введении. При этом будет использован следующий результат, принадлежащий Мальцеву [3]:

Предложение 14. Всякая связная подгруппа Ли односвязной разрешимой группы Ли также односвязна.

Доказательство. Воспользуемся индукцией по размерности группы. Пусть $G$ - односвязная разрешимая группа Ли, а $H$ - ее связная подгруппа. Заметим, что коммутант $(G, G)$ односвязен. Действительно, $G /(G, G)$ - односвязная коммутативная группа, так что $\pi_{2}(G /(G, G))=0$. Тогда из точной последовательности

$$
\pi_{2}(G /(G, G)) \longrightarrow \pi_{1}((G, G)) \longrightarrow \pi_{1}(G)
$$

следует, что коммутант односвязен. Положим $H_{1}=H \cap(G, G)$ и рассмотрим точную последовательность

$$
\pi_{1}\left(H_{1}\right) \longrightarrow \pi_{1}(H) \longrightarrow \pi_{1}\left(H / H_{1}\right) \longrightarrow \pi_{0}\left(H_{1}\right) \longrightarrow \pi_{0}(H)=0 .
$$

Факторгруппа $H / H_{1}$ односвязна как подгруппа векторной группы. Следовательно, подгруппа $H_{1}$ связна и даже односвязна по предположению индукции. Поэтому подгруппа $H$ односвязна.

Лемма 15. Пусть $G \cong C 入 N$, где $C$ и $N$ - связные $\sigma$-инвариантные подгруппы, $C \cong \mathbb{R}^{n}$ и $N \subset M$. Тогда множество всех антиинвариантных элементов группы $G$ свлзно.

Доказательство. Пусть $g=c n$, где $c \in C, n \in N$. Тогда $g \sigma(g)=c n \sigma(c) n^{-1}=$ $(\mathrm{id}+a(n) \sigma)(c)$, где $a(n) c=n c n^{-1}$. Таким образом, для каждого элемента $n \in N$ множество антиинвариантных элементов в смежном классе $C n$ связно, так как оно имеет вид $V n$, где $V \subset C$ - линейное подпространство. Но подгруппа $N$ связна и состоит из антиинвариантных элементов. Следовательно, подмножество антиинвариантных элементов группы $G$ связно.

Доказательство теоремы 1. Достаточно рассмотреть случай, когда группа $G$ односвязна. Воспользуемся индукцией по размерности группы. Пусть $N$ - минимальная $\sigma$-инвариантная нормальная подгруппа группы $G$. Заметим, что подгруппа $N$ обязана быть коммутативной, так как в противном случае коммутант $(N, N)$ был бы меньшей связной $\sigma$-инвариантной нормальной подгруппой. Кроме того, подгруппа $N$ односвязна по предложению 14 . Положим 
$\widehat{G}=G / N$ и обозначим через $\pi: G \rightarrow \widehat{G}$ отображение факторизации. По предположению индукции пространство $\widehat{G} / \widehat{G}^{\sigma}$ слабо экспоненциально, а значит, $\pi(M)=M_{\widehat{G}}=\exp _{\widehat{G}} \hat{\mathfrak{m}}$. Пусть $U \subset M$ - некоторое открытое подмножество. Найдется такой $\xi \in \hat{\mathfrak{m}}$, что $\exp _{\widehat{G}} \xi \in \pi(U)$. Обозначим через $F \subset G$ полный прообраз однопараметрической группы, порожденной элементом $\xi$. Это $\sigma$-инвариантная подгруппа, изоморфная полупрямому произведению $N \lambda \exp \left\langle\xi^{\prime}\right\rangle$, где $\xi^{\prime} \in \mathfrak{m}$, $\mathrm{d} \pi\left(\xi^{\prime}\right)=\xi$. По лемме 15 подмножество антиинвариантных элементов группы $F$ связно. Достаточно доказать, что $F \cap M=\overline{\exp (\mathfrak{f} \cap \mathfrak{m})}$. Но это условие эквивалентно слабой экспоненциальности симметрического пространства $F / F^{\sigma}$, так как $F \cap M=M_{F}$. Теперь уточним структуру подгруппы $F$.

Пусть $N_{0}$ - минимальная нормальная (не обязательно $\sigma$-инвариантная) подгруппа Ли группы $G$, содержащаяся в $N$. Алгебра $\mathfrak{n}_{0}=$ Lie $N_{0}$ является неприводимым g-модулем и, следовательно, имеет размерность 1 или 2 . Группа $\sigma\left(N_{0}\right)$ также является минимальной нормальной подгруппой Ли группы $G$, причем $\sigma$ устанавливает изоморфизм $N_{0} \cong \sigma\left(N_{0}\right)$. Подгруппы $N_{0}$ и $\sigma\left(N_{0}\right)$ либо совпадают (и тогда $N=N_{0}$ ), либо пересекаются по некоторой дискретной подгруппе. Группа $N$ односвязна, так что в последнем случае она изоморфна $N_{0} \times \sigma\left(N_{0}\right)$. Если оператор ad $\left.\xi^{\prime}\right|_{\mathfrak{n}}$ не имеет чисто мнимых собственных значений, то группа $F$ сильно экспоненциальна и, следовательно, пространство $F / F^{\sigma}$ экспоненциально. Таким образом, можно считать, что $\operatorname{dim} N_{0}=2$, и доказательство теоремы сводится к рассмотрению двух случаев:

1) $N=N_{0}$. В этом случае пространство $F / F^{\sigma}$ изоморфно пространству, рассмотренному в примере 1 . Оно слабо экспоненциально.

2) $N=N_{0} \times N_{0}$. В этом случае $\mathfrak{f}=\mathbb{R}+(\mathbb{C} \oplus \mathbb{C}), \mathfrak{n}=\mathbb{C} \oplus \mathbb{C}, \xi^{\prime}=(1,0,0)$, $\operatorname{ad}(\xi)(t, u, v)=(0, i u,-i v), \sigma(t, u, v)=(-t, v, u)$. Тогда множество $\mathfrak{n}^{\prime}=\{(t, u, \bar{u}):$ $t \in \mathbb{R}, u \in \mathbb{C}\}$ является $\sigma$-инвариантным идеалом в алгебре f. Пусть $N^{\prime} \subset F$ - подгруппа, соответствующая подалгебре $\mathfrak{n}^{\prime}$. Тогда $N^{\prime}$ есть $\sigma$-инвариантная нормальная подгруппа группы $F$. Повторение тех же рассуждений, что и в начале доказательства, сводит ситуацию к случаю 1.

Теорема 16. Пусть $N$ - связная $\sigma$-инвариантная нормальная подгруппа группь $G$ и $\widehat{G}=G / N$. Тогда пространства $G / G^{\sigma}$ и $\widehat{G} / \widehat{G}^{\sigma}$ слабо экспоненциальны или не слабо экспоненииальны одновременно.

Доказательство. Пусть пространство $\widehat{G} / \widehat{G}^{\sigma}$ слабо экспоненциально. Докажем слабую экспоненциальность пространства $G / G^{\sigma}$ с помощью индукции по $\operatorname{dim} N$.

Пусть группа $N$ коммутативна. Тогда $N \cong \mathbb{R}^{k} \times \mathbb{T}^{l}$, причем обе компоненты прямого произведения являются $\sigma$-инвариантными нормальными подгруппами группы $G$. Поэтому достаточно рассмотреть случаи, когда $N \cong \mathbb{R}^{k}$ или $N \cong \mathbb{T}^{l}$.

Рассмотрим некоторое открытое подмножество $U \subseteq M$. Множество $\pi(U)$ открыто в $\widehat{M}$, так как $\pi(M)=\widehat{M}$. Следовательно, существует такой элемент $\hat{\xi} \in \hat{\mathfrak{m}}$, что $\exp \hat{\xi} \in \pi(U)$. Пусть $F-$ полный прообраз однопараметрической подгруппы, порожденной вектором $\hat{\xi}$, при отображении $\pi$. Докажем, что $\exp (\mathfrak{f} \cap$ $\mathfrak{m}) \cap U \neq \varnothing$. Так как группа $F$ разрешима, то множество $\exp (\mathfrak{f} \cap \mathfrak{m})$ всюду плотно в $(F \cap M)^{0}$, так что для этого достаточно доказать связность пересечения $F \cap M$. В случае когда $N \cong \mathbb{R}^{k}$, это следует из леммы 15 . Если же $N \cong \mathbb{T}^{l}$, то $N \in Z(G)$. При этом $N^{\sigma}$ лежит в ядре неэффективности действия $G: G / G^{\sigma}$, так что можно считать, что $\left.\sigma\right|_{N}$ - инверсия. Тогда $F \subset M$. 
В общем случае обозначим через $C$ последний нетривиальный член ряда коммутантов группы $N$. Это связная $\sigma$-инвариантная нормальная подгруппа группы $G$. Положим $G_{1}=G / C$. Тогда $\widehat{G}=G_{1} /(N / C)$ и пространство $G_{1} / G_{1}^{\sigma}$ слабо экспоненциально по предположению индукции. Но группа $C$ коммутативна. Следовательно, пространство $G / G^{\sigma}$ также экспоненциально.

Теперь, чтобы получить утверждение теоремы 2 , нужно в условии последней теоремы положить $N=\operatorname{Rad}(G)$.

\section{§4. Критерий слабой экспоненциальности}

При изучении экспоненциального отображения групп Ли важную роль играют картановские подгруппы и регулярные элементы. Для доказательства критерия слабой экспоненциальности нам необходимо обобщение этих понятий на симметрические пространства, впервые введенное Картаном [6]. Определение картановской группы симметрического пространства дано нами в 1 .

Пусть $\mathfrak{g}$ - редуктивная линейная алгебра Ли. Назовем элемент $\xi \in \mathfrak{m} p e-$ гулярным в $\mathfrak{m}$, если размерность его централизатора в $\mathfrak{m}$ минимальна. Аналогично, элемент $g \in M$ назовем регулярным в $M$, если размерность его централизатора в $\mathfrak{m}$ минимальна. Обозначим множества элементов, регулярных соответственно в $\mathfrak{m}$ и в $M$, через $\mathfrak{m}^{\text {reg }}$ и $M^{\text {reg }}$. Это открытые и всюду плотные подмножества соответственно в $\mathfrak{m}$ и в $M$.

Следующее утверждение доказано в [13] (следствие леммы 4):

Предложение 17. Пусть $G$ - редуктивная линейная группа Ли и $\mathfrak{a}$ - картановская алгебра пространства $G / G^{\sigma}$. Тогда в а найдется такой регулярный в $\mathfrak{m}$ элемент $\xi$, что $\mathfrak{z}_{\mathfrak{m}}(\xi)=\mathfrak{z}_{\mathfrak{m}}(\mathfrak{a})=\mathfrak{a}$.

Лемма 18. Пусть группа Ли $G$ произвольна, $\xi \in \mathfrak{m} u g=\exp \xi$ таков, что размерность иентрализатора $\mathfrak{z}_{\mathfrak{m}}(g)$ минимальна. Тогда $\mathfrak{z}_{\mathfrak{m}}(\xi)=\mathfrak{z}_{\mathfrak{m}}(g)$.

Доказательство. Так как $\operatorname{Ad}(\exp t \xi)=\exp (\operatorname{ad} t \xi)$, то для любого $t \in \mathbb{R}$ верно включение $\mathfrak{z}_{\mathfrak{m}}(\xi) \subseteq \mathfrak{z} \mathfrak{m}(\exp t \xi)$, причем найдется такое число $\epsilon$, что при $|t|<\epsilon$ это включение обращается в равенство. Так как размерность пространства $\mathfrak{z m}(g)$ минимальна, из этого следует, что $\mathfrak{z} \mathfrak{m}(\xi)=\mathfrak{z}_{\mathfrak{m}}(g)$.

Пусть $G$ - редуктивная линейная группа Ли. Из леммы 18 следует, что если элемент $g=\exp \xi$ регулярен в $M$, то $\xi$ регулярен в $\mathfrak{m}$. Если при этом элемент $g$ полупрост, то элемент $\xi$ также полупрост, а подпространство $\mathfrak{z} \mathfrak{m}(g)=\mathfrak{z m}(\xi)$ является картановской алгеброй пространства $G / G^{\sigma}$. Мы будем обозначать эту алгебру через $\mathfrak{a}(g)$, а связную компоненту единицы соответствующей картановской группы - через $A(g)$. Так как $\mathfrak{a}(g)$ коммутативна, то $A(g)=\exp \mathfrak{a}(g)$. Из леммы 18 следует, что если $g \in \exp \mathfrak{m}$, то $g \in A(g)$.

Лемма 19. Пусть $G$ - редуктивная линейная группа Ли. Фиксируем полупростой регулярный в $M$ элемент g и окрестность единицы $W \subseteq G^{\sigma}$. Cyществует такая окрестность $V \subseteq M$ элемента $g$, что для любого полупростого регулярного в $M$ элемента $g^{\prime} \in V$ найдется такой $h \in W$, что $\operatorname{Ad}(h) \mathfrak{a}\left(g^{\prime}\right)=\mathfrak{a}(g)$.

Доказательство. Обозначим через $A$ картановскую группу, соответствующую $\mathfrak{a}(g)$. Так как множество полупростых регулярных элементов открыто в $M$, то существует окрестность $U \subset A$ элемента $g$, каждый элемент которой регулярен и полупрост. Тогда $\mathfrak{a}\left(g^{\prime}\right)=\mathfrak{a}(g)$ для каждого $g^{\prime} \in U$. Пусть 
$V \subset \operatorname{Ad}(W)(U) \subset M$ - окрестность элемента $g$. Выбрав окрестность достаточно малой, можно считать, что $V$ - подмногообразие в $M$, имеющее размерность $\operatorname{dim} \mathfrak{a}+\operatorname{dim} \mathfrak{h}-\operatorname{dim} \mathfrak{z} \mathfrak{h}(\mathfrak{a})$. Пусть $\mathfrak{b} \subset \mathfrak{m}-$ какое-то подпространство, дополнительное к $\mathfrak{z} \mathfrak{h}(\mathfrak{a})$. Тогда размерность $V$ равна $\operatorname{dim} \mathfrak{a}+\operatorname{dim} \mathfrak{b}$. Пусть $\zeta \in \mathfrak{a}-$ полупростой регулярный элемент. Тогда $\mathfrak{z} g(\zeta)=\mathfrak{z} \mathfrak{g}(\mathfrak{a})$, и $\operatorname{ad}(\zeta)$ взаимно однозначно отображает $\mathfrak{b}$ на некоторое подпространство $\mathfrak{c} \subset \mathfrak{m}$, дополнительное к $\mathfrak{a}$. Таким образом, $\operatorname{dim} V=\operatorname{dim} M$. Следовательно, $V$ открыто в $M$. При этом если $g^{\prime} \in V$, то $\mathfrak{a}\left(g^{\prime}\right)=\operatorname{Ad}(h) \mathfrak{a}(g)$.

Теорема 20. Пусть $G$ - редуктивная линейная группа Ли. Пространство $G / G^{\sigma}$ слабо экспоненциально тогда и только тогда, когда все его картановские группы связны.

Доказательство. Пусть пространство $G / G^{\sigma}$ слабо экспоненциально и $g-$ полупростой элемент, регулярный в $M$. Покажем, что $g \in \exp \mathfrak{m}$. Выберем окрестность $V$, как в лемме 19. Найдется полупростой регулярный элемент $g^{\prime} \in$ $V \cap \exp \mathfrak{m}$. Но тогда существует такой $h \in G^{\sigma}$, что $g=\operatorname{Ad}(h) g^{\prime} \in \operatorname{Ad}(h) \exp \mathfrak{a}\left(g^{\prime}\right)=$ $\exp \mathfrak{a}(g)$. Теперь пусть $A$ - произвольная картановская группа. Рассмотрим элемент $g \in A$ и некоторую его окрестность $U$, целиком лежащую в той же компоненте связности группы $A$. Найдется некоторый полупростой элемент $g_{1} \in M^{\text {reg }} \cap U, g_{1}=\exp \xi$. При этом $\mathfrak{a}=\operatorname{Lie} A=\mathfrak{a}\left(g_{1}\right)$. Но тогда $\xi \in \mathfrak{a}$ по лемме 18. Получаем, что $g_{1} \in A^{0}$.

Обратно, пусть любая картановская группа связна. Любой полупростой элемент $g \in M^{\text {reg }}$ лежит в картановской группе $A=Z_{M}(\mathfrak{a}(g))$. Группа $A$ связна и коммутативна, а значит, экспоненциальна, $g \in A=\exp \mathfrak{a}(g) \subset \exp \mathfrak{m}$. Множество полупростых регулярных в $M$ элементов плотно в $M$ и содержится в множестве exp $\mathfrak{m}$. Следовательно, $\overline{\exp \mathfrak{m}}=M$ и пространство $G / G^{\sigma}$ слабо экспоненциально.

Следствие 21. Пусть $G-$ связная комплексная группа Ли и $\sigma-$ ее инволютивный комплексно-аналитический автоморфизм. Тогда пространство $G / G^{\sigma}$ слабо экспоненциально.

Доказательство. Воспользовавшись теоремой 2 и предложением 13, можно свести утверждение к случаю, когда $G$ - односвязная полупростая алгебраическая группа. Но тогда все картановские группы связны [13, лемма 8].

Таким образом, для проверки слабой экспоненциальности заданного аффинного симметрического пространства необходимо исследовать связность всех его картановских групп. Задача сводится к исследованию классов картановских групп, сопряженных относительно $G^{\sigma}$. Далее мы покажем, что достаточно рассматривать только один класс сопряженности.

Фиксируем инволюцию Картана $\theta$, коммутирующую с автоморфизмом $\sigma[5]$, и соответствующее разложение Картана $\mathfrak{g}=\mathfrak{k}+\mathfrak{p}$, где $\mathfrak{k}=\mathfrak{g}^{\theta}, \mathfrak{p}=\{\xi \in \mathfrak{g}: \theta(\xi)=$ $-\xi\}$. Обозначим через $K$ связную подгруппу группы $G$, соответствующую подалгебре $\mathfrak{k}$.

Следующие факты доказаны в работах [11] и [14].

Предложение 22. (а) [14, теорема 1] Любая картановская алгебра сопряжена $\theta$-инвариантной картановской алгебре.

(b) [11, лемма 8] Пусть картановские алгебры $\mathfrak{a}_{1} u \mathfrak{a}_{2} \theta$-инвариантны, причем $\operatorname{dim}\left(\mathfrak{a}_{1} \cap \mathfrak{k}\right) \leqslant \operatorname{dim}\left(\mathfrak{a}_{2} \cap \mathfrak{k}\right)$. Тогда найдется такой $g \in G^{\sigma}$, что для картановской алгебры $\mathfrak{a}_{1}^{\prime}=\operatorname{Ad}(g) \mathfrak{a}_{1}$ выполнены включения $\mathfrak{a}_{1}^{\prime} \cap \mathfrak{k} \subseteq \mathfrak{a}_{2} \cap \mathfrak{k}, \mathfrak{a}_{2} \cap \mathfrak{p} \subseteq \mathfrak{a}_{1}^{\prime} \cap \mathfrak{p}$. 
(с) [11, лемма 7] Любъе две максимально некомпактные картановские группъ сопряжены при помощи $G^{\sigma}$.

Пусть а есть $\theta$-инвариантная картановская алгебра и $A$ - соответствующая картановская группа. Обозначим через $\mathfrak{g}(\mathbb{C})$ комплексификацию алгебры $\mathfrak{g}$ и рассмотрим односвязные группы $G(\mathbb{C})$ и $\widetilde{G}$, соответствующие этим касательным алгебрам. Пусть $j: \widetilde{G} \rightarrow G(\mathbb{C})$ - вложение, а $\pi: \widetilde{G} \rightarrow G$ - каноническая проекция.

Предложение 23. Пусть а есть $\theta$-инвариантная картановская алгебра и $A$ - соответствующая картановская группа. Тогда $A=C_{A} \exp \mathfrak{a}$, где $C_{A}=$ $\pi\left(\exp (i \operatorname{ad}(\mathfrak{a} \cap \mathfrak{p})) \cap M_{\widetilde{G}}\right)$.

Доказательство. Так как $A=(A \cap K) \exp (\mathfrak{a} \cap \mathfrak{p})$, достаточно доказать, что $A \cap K=C_{A} \exp (\mathfrak{a} \cap \mathfrak{k})$. Включение $C_{A} \exp (\mathfrak{a} \cap \mathfrak{k}) \subset A \cap K$ очевидно.

Обозначим через $\mathfrak{a}(\mathbb{C}) \subset \mathfrak{g}(\mathbb{C})$ комплексификацию алгебры $\mathfrak{a}$. Алгебра $\mathfrak{a}(\mathbb{C})$ будет картановской алгеброй пространства $G(\mathbb{C}) / G(\mathbb{C})^{\sigma}$, а соответствующая картановская группа $A(\mathbb{C})$ будет связной, как и всякая картановская группа симметрического пространства комплексной группы Ли. Тогда $A \cap K \subset$ $\pi(\exp (\mathfrak{a}(\mathbb{C})) \cap \widehat{G})$. Более того, $A \cap K \subset \pi(\exp ((\mathfrak{a} \cap \mathfrak{k})+i(\mathfrak{a} \cap \mathfrak{p})) \cap \widehat{G})$. Следовательно, $A \cap K \subset \pi\left(\exp (\mathfrak{a} \cap \mathfrak{k}) \exp (i(\mathfrak{a} \cap \mathfrak{p})) \cap M_{\widetilde{G}}\right)=\exp (\mathfrak{a} \cap \mathfrak{k}) C_{A}$, что и требовалось доказать.

Из предложения 23 следует, что $\theta$-инвариантная картановская группа $A$ связна тогда и только тогда, когда $C_{A} \subset \exp (\mathfrak{a} \cap \mathfrak{k})$.

Доказательство теоремы 3. Эквивалентность условий 1 и 2 уже доказана в теореме 20. Докажем эквивалентность условий 2 и 3.

Пусть максимально некомпактная картановская группа $A_{0}$ связна, $\mathfrak{a}_{0}=$ Lie $A_{0}, \mathfrak{a}$ - произвольная картановская алгебра пространства $G / G^{\sigma}$ и $A-$ соответствующая группа. Воспользовавшись предложением 22 , можно считать, что алгебра $\mathfrak{a} \theta$-инвариантна и $\mathfrak{a}_{0} \cap \mathfrak{k} \subseteq \mathfrak{a}_{0} \cap \mathfrak{k}, \mathfrak{a} \cap \mathfrak{p} \subseteq \mathfrak{a}_{0} \cap \mathfrak{p}$. Тогда $C_{A}=$ $\operatorname{Ad}^{-1}(\exp (i \operatorname{ad}(\mathfrak{a} \cap \mathfrak{p})) \cap \operatorname{Ad}(K)) \subseteq C_{A_{0}} \subset \exp \left(\mathfrak{a}_{0} \cap \mathfrak{k}\right) \subseteq(\mathfrak{a} \cap \mathfrak{k})$. Следовательно, группа $A$ связна. Обратное утверждение очевидно.

Замечание. Эта теорема обобщает результат для групп Ли, полученный Неебом [12, теорема II.2].

Пример 2. Пусть $G=\mathrm{SU}(1,1), \sigma(g)=\bar{g}, G^{\sigma}=\mathrm{SO}(1,1)$. Тогда $\mathfrak{m}=\left\{\left(\begin{array}{cc}i x & i y \\ -i y & -i x\end{array}\right)\right.$ : $x, y \in \mathbb{R}\}, \mathfrak{m} \cap \mathfrak{p}=\mathfrak{a}_{0}=\left\{\left(\begin{array}{cc}0 & i y \\ -i y & 0\end{array}\right): y \in \mathbb{R}\right\}, M=\left\{\left(\begin{array}{cc}a+i b & i c \\ -i c & a-i b\end{array}\right): a, b, c \in \mathbb{R}\right.$, $\left.a^{2}+b^{2}-c^{2}=1\right\}$. Централизатор $Z_{G}\left(\mathfrak{a}_{0}\right)=\left\{\left(\begin{array}{cc}a & i c \\ -i c & a\end{array}\right): a, c \in \mathbb{R}, a^{2}-c^{2}=1\right\}$ целиком содержится в $M$. Он состоит из двух компонент связности, и поэтому пространство $G / G^{\sigma}$ не слабо экспоненциально. Однако централизатор алгебры $\mathfrak{a}_{0}$ в группе $G_{1}=\operatorname{PSU}(1,1)$ связен, так что пространство $G_{1} / G_{1}^{\sigma}$ (здесь $G_{1}^{\sigma}=\operatorname{PSO}(1,1) \lambda\left\langle g_{0}\right\rangle_{2}$, где $\left.g_{0}=\left(\begin{array}{cc}0 & 1 \\ -1 & 0\end{array}\right)\right)$ слабо экспоненциально.

\section{§5. Критерий экспоненциальности}

В этом параграфе будет доказан критерий экспоненциальности для линейных расщепимых групп Ли (теорема 4). Напомним, что линейная группа Ли называется расщепимой, если содержит компоненты разложения Жордана каждого своего элемента. 
Предложение 24. Пусть $L-$ линейная расщепимая группа Ли, $\sigma-$ ее инволютивный автоморфизм и $g \in L-$ полупростой элемент, $\sigma(g)=g^{-1}$. Тогда существует $\sigma$-инвариантная редуктивная подгруппа Леви $S$, содержсащая $g$.

Доказательство. Рассмотрим случай, когда унипотентный радикал $U$ группы $L$ коммутативен. Множество всех подгрупп Леви есть однородное пространство группы $U$. Пусть $S$ - подгруппа Леви, содержащая элемент $g$, и $g \in$ $\sigma(S)=u S u^{-1}$, где $u \in U$. Так как подгруппа $U$ нормальна в $L$, а $u^{-1} g u \in S$, то $g^{-1} u^{-1} g u \in U \cap S=\{e\}$. Поэтому множество подгрупп Леви, содержащих элемент $g$, есть орбита подгруппы $Z_{U}(g) \subset U$. Автоморфизм $\sigma$ индуцирует ее инволютивное аффинное преобразование, для которого имеется неподвижная точка. Соответствующая редуктивная подгруппа Леви будет $\sigma$-инвариантной.

Теперь утверждение нашей теоремы может быть доказано с помощью индукции по длине центрального ряда группы $U$. Пусть $V$ - последний ненулевой член центрального ряда группы $U$. Это $\sigma$-инвариантная нормальная подгруппа группы $L$. В факторгруппе $L / V$ найдется $\sigma$-инвариантная редуктивная группа Леви $S_{0}$, содержащая образ элемента $g$ при канонической проекции $\pi: L \rightarrow L / V$, который также является полупростым. Полный прообраз этой подгруппы $\pi^{-1}\left(S_{0}\right)$ будет $\sigma$-инвариантной подгруппой группы $L$ с коммутативным унипотентным радикалом. Любая $\sigma$-инвариантная подгруппа Леви в $\pi^{-1}\left(S_{0}\right)$ является искомой подгруппой Леви в $L$.

Следствие 25. Пусть симметрическое пространство $G / G^{\sigma}$ расщепимой группы Ли $G$ слабо экспоненицально. Тогда любой полупростой элемент $g \in M$ лежит в ехр $\mathfrak{m}$.

Доказательство. Всякий полупростой элемент $g \in M$ лежит в некоторой $\sigma$-инвариантной подгруппе Леви $L \subset G$. Следовательно, $g$ содержится в некоторой картановской группе $A$ пространства $L / L^{\sigma}$. Это пространство слабо экспоненциально по теореме 16 , а значит, группа $A$ связна. Так как она коммутативна, то $g \in A=\exp \mathfrak{a} \subset \exp \mathfrak{m}$.

Доказательство теоремы 4. Пусть пространство $G / G^{\sigma}$ экспоненциально и $\xi \in \mathfrak{m}-$ нильпотентный элемент. Рассмотрим произвольный элемент $g \in Z_{M}(\xi)$ и его разложение Жордана $g=g_{s} g_{u}$, где $g_{s}$ и $g_{u}$ - полупростая и унипотентная компоненты соответственно. Из единственности разложения Жордана следует, что $\sigma\left(g_{u}\right)=g_{u}^{-1}, \sigma\left(g_{s}\right)=g_{s}^{-1}$. Нильпотентный элемент $\xi_{n}=\log \left(g_{u}\right)$ лежит в $\mathfrak{z}_{\mathfrak{m}}(\xi)$, а значит, $g_{u}=\exp \xi_{n} \in M$. Элементы $g$ и $g_{s}$ можно соединить непрерывным путем $\gamma(t)=g_{s} \exp t \xi_{n}$, каждая точка которого является антиинвариантным элементом, коммутирующим с $\xi$. Следовательно, $g_{s} \in M$. Для любого $t \in \mathbb{R}$ антиинвариантные элементы $g_{s}$ и $\exp t \xi$ коммутируют, так что их произведение также является антиинвариантом. Таким образом, элемент $g^{\prime}=g_{s} \exp \xi$ лежит в $M$, причем $g_{s}$ и $\exp \xi$ являются соответственно полупростой и унипотентной компонентами его разложения Жордана. Так как $M=\exp \mathfrak{m}$, то существует такой $\eta \in \mathfrak{m}$, что $g^{\prime}=\exp \eta$. Рассмотрим его разложение Жордана $\eta=\eta_{s}+\eta_{n}$, где $\eta_{s}-$ полупростой, а $\eta_{n}-$ нильпотентный элементы. Тогда $g^{\prime}=\exp \eta_{s} \exp \eta_{n}-$ разложение Жордана элемента $g^{\prime}$. Из единственности такого разложения следуют равенства $g_{s}=\exp \eta_{s}, \exp \xi=\exp \eta_{n}$. Но тогда $\xi=\eta_{n}$ и $g_{s} \in \exp \mathfrak{z m}(\xi)$.

Мы получили, что произвольный элемент $g \in Z_{M}(\xi)$ можно представить в виде $g=\exp \eta_{s} \exp \eta_{n}$, где $\eta_{s}, \eta_{n} \in \mathfrak{z}_{\mathfrak{m}}(\xi)$. Следовательно, множество $Z_{M}(\xi)$ связно. Теперь рассмотрим подмножество элементов в $Z_{M}(\xi)$, у которых размерность 
централизатора полупростой части в $\mathfrak{m}$ минимальна. Это подмножество всюду плотно в $Z_{M}(\xi)$, и по лемме 18 для любого такого элемента $g$ выполнено равенство $\mathfrak{z}_{\mathfrak{m}}\left(g_{s}\right)=\mathfrak{z}_{\mathfrak{m}}\left(\eta_{s}\right)$. Это означает, что $g=\exp \left(\eta_{s}\right) \exp \left(\eta_{n}\right)=\exp \left(\eta_{s}+\eta_{n}\right) \in$ $\exp \mathfrak{m}$, так как $\left[\eta_{s}, \eta_{n}\right]=0$. Следовательно, $Z_{M}(\xi)=\overline{\operatorname{exp~} \mathfrak{z} \mathfrak{m}(\xi)}$.

Теперь пусть для любого нильпотентного элемента $\xi \in \mathfrak{m}$ выполнено условие $Z_{M}(\xi)=\overline{\exp \mathfrak{z m}(\xi)}$. Докажем, что пространство $G / G^{\sigma}$ экспоненциально. Пусть $g$ - элемент из $M$ и $g=g_{s} g_{u}$ - его разложение Жордана. Существует такой нильпотентный элемент $\xi_{n} \in \mathfrak{m}$, что $g_{u}=\exp \xi_{n}$. Рассмотрим группу $Z_{G}\left(\xi_{n}\right)$. Это $\sigma$-инвариантная алгебраическая подгруппа группы $G$, содержащая $g_{s}$. Обозначим через $Z$ ее связную компоненту, содержащую единицу. По условию $Z \cap M=\overline{\exp (\mathfrak{z} \cap \mathfrak{m})}$, т. е. пространство $Z / Z^{\sigma}$ слабо экспоненциально. Так как $g_{s} \in Z \cap M$, то по следствию 25 существует такой элемент $\xi_{s} \in \mathfrak{z}_{\mathfrak{m}}\left(\xi_{n}\right)$, что $g_{s}=\exp \xi_{s}$. Но тогда $g=\exp \left(\xi_{s}+\xi_{n}\right) \in \exp \mathfrak{m}$, что и требовалось доказать.

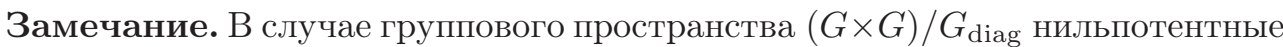
элементы в $\mathfrak{m}$ имеют вид $(\xi,-\xi)$, где $\xi$ - нильпотентный элемент в $\mathfrak{g}$. Положим $Z=Z_{G}(\xi)$. Тогда $Z_{G \times G}(\xi,-\xi) \cong Z \times Z$. Связность множества $Z_{G \times G}(\xi,-\xi) \cap M=$ $\left\{\left(g, g^{-1}\right): g \in Z\right\}$ эквивалентна связности группы $Z$. Кроме того, как мы уже отмечали во введении, слабая экспоненциальность пространства $(Z \times Z) / Z_{\text {diag }}$ эквивалентна слабой экспоненциальности группы $Z$. Таким образом, теорема 4 является обобщением критерия экспоненциальности для расщепимых групп Ли, полученного Вюстнером [15], который состоит в том, что связная группа Ли $G$ экспоненциальна тогда и только тогда, когда для любого нильпотентного элемента $\xi \in \mathfrak{g}$ его централизатор $Z=Z_{G}(\xi)$ слабо экспоненциален.

Пример 3. Пусть $G=\mathrm{SU}(p, p), \sigma(g)=\bar{g}, G^{\sigma}=\mathrm{SO}(p, p)$. Классы сопряженных нильпотентных элементов алгебры $\mathfrak{g}=\mathfrak{s u}(p, p)$ параметризуются отмеченными диаграммами Юнга сигнатуры $(p, p)$, причем представитель в $\mathfrak{m}$ имеется у тех классов, в диаграммах которых для любого целого $n$ имеется четное число строк длины $2 n$. В частности, в $\mathfrak{m}$ лежит нильпотентный элемент $\eta$, соответствующий диаграмме из одной строки длины $2 p$. Представление алгебры $\mathfrak{s l}(2, \mathbb{R})$, связанное с этим элементом, неприводимо. Поэтому редуктивная часть централизатора $Z_{G}(\eta)$, совпадающая с централизатором всей $\mathfrak{s l}(2, \mathbb{R})$-тройки, совпадает с центром группы $G$. Но центр группы $G$ лежит в $M$ и даже в множестве $\exp \mathfrak{m}$, так как $\operatorname{diag}\left(e^{\pi i k / p}, \ldots, e^{\pi i k / p}\right)=\exp (\pi i \operatorname{diag}(k / p, \ldots, k / p, k / p-2 k)$. Таким образом, число компонент связности множества $Z_{M}(\eta)$ равно порядку центра группы $G$. Следовательно, пространство $\mathrm{SU}(p, p) / \mathrm{SO}(p, p)$ не экспоненциально.

\section{ЛитерАтУРА}

[1] Э. Б. Винберг, В. В. Горбацевич, А. Л. Онищик, Строение групn и алгебр Ли, в кн.: Итоги науки и техники. Современные проблемы математики. Фундаментальные направления, т. 41, ВИНИТИ, М., 1990, 5-258.

[2] Э. Б. Винберг, В. Л. Попов, Теория инвариантов, в кн.: Алгебраическая геометрия-4, Итоги науки и техники. Современные проблемы математики. Фундаментальные направления, т. 55, ВИНИТИ, М., 1989, 137-309.

[3] А. И. Мальцев, On the theory of the Lie groups in the large [K meopuu гpynn Лu 8 целом], Матем. сб., 16:2 (1945), 163-189; 19:3 (1946), 523-524.

[4] К. Шевалле, Теория групп Ли, т. 3, ИЛ, М., 1958. 
[5] M. Berger, Les espaces symétriques non compacts, Ann. Sci. École Norm. Sup., 74 (1957), 85-177.

[6] E. Cartan, Sur certains formes Riemanniennes remarquables des géométries à groupe foundamental simple, Ann. Sci. École Norm. Sup., 44 (1927), 345-467.

[7] D. Z̆. Djoković, K. H. Hofmann, The surjectivity question for the exponential function of real Lie groups: A status report, J. Lie Theory, 7 (1997), 171-199.

[8] K. H. Hofmann, A. Mukherjea, On the density of the image of the exponential function, Math. Ann., 234 (1978), 263-273.

[9] K. H. Hofmann, A memo on the exponential function and regular points, Arch. Math., 59 (1992), 24-37.

[10] A. L. Konstantinov, P. K. Rozanov, On the exponential map of the Lie groups locally isomorphic to $S U(p, q)$, J. Lie Theory, 15 (2005), 51-61.

[11] T. Matsuki, The orbits of affine symmetric spaces under the action of minimal parabolic subgroups, J. Math. Soc. Japan, 31:2 (1979), 331-357.

[12] K.-H. Neeb, Weakly exponential Lie groups, J. Algebra, 179:2 (1996), 331-361.

[13] T. Oshima, T. Matsuki, Orbits on affine symmetric spaces under the action of the isotropy subgroups, J. Math. Soc. Japan, 32:2 (1980), 399-414.

[14] W. Rossmann, The structure of semisimple symmetric spaces, Canad. J. Math., 31:1 (1979), 157-180.

[15] M. Wüstner, On the exponential function of real splittable and real semisimple Lie groups, Beiträge Algebra Geom., 39:1 (1998), 37-46.

[16] M. Wüstner, The classification of all simple Lie groups with surjective exponential map, J. Lie Theory, 15:1 (2005), 269-278.

Московский государственный университет e-mail: p.k.rozanov@gmail.com

Поступило в редакцию 6 июня 2007 г. 